electrode. Adding to the 50 c.c. of $0.002 \mathrm{~N}$ potassium chloride an emulsion of $4 \mathrm{gm}$. terpineol and $4 \mathrm{gm}$. potassium xantogenicum in 1000 c.c. of $0.002 \mathrm{~N}$ potassium chloride of the same $p \mathrm{H}$, namely, $7 \cdot 5$, there was found a remarkable difference in the behaviour between the unattackable electrodes, platinum, galena, and graphite and the attackable zinc. Zinc changes its potential very little; the indifferent electrodes mentioned above, however, show a very marked fall of the potential as is shown in Fig. 1.

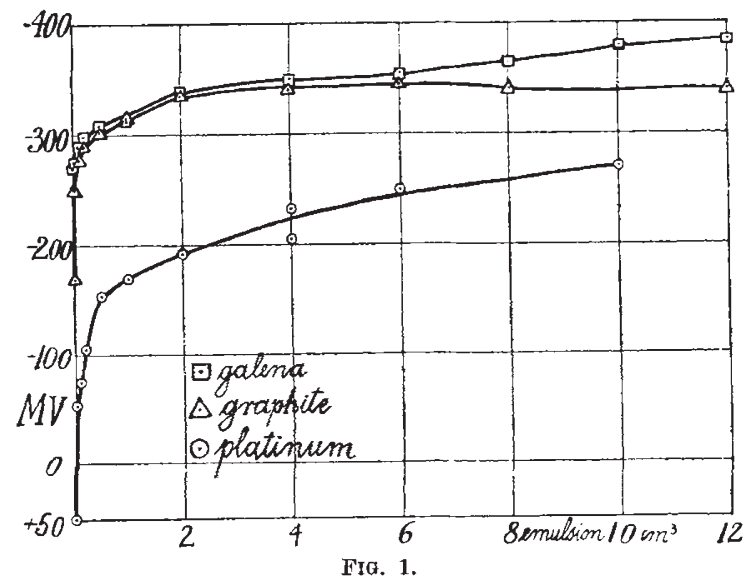

The first portion of 0.02 c.c. of the terpineol and potassium xantogenicum emulsion has a great influence on the potential of the electrode in spite of the fact that the emulsion loses its charucter at that dilution, and at 1 c.c. of these weak solutions has a maximum effect. Solutions of that strength are used in practice for flotation most frequently. It should be added that the electrometric measuring of the hydrogen ion concentration of physiological yeasts may be entirely spoiled if such a minute concentration as 1 in 500,000 by weight of an organic compound changes the potential of platinum so markedly.

Institute of Physical Chemistry,

\section{B. KAMIENSKI.}

Politechnika, Lwow,

Nov. 17.

1 Freundlich, H., Ettisch, G., Zeit. für phys. Chem., 116, 401-419; 1925.

Kamienski, B., Zeit. für phys. Chem., 138, 345 ; 1928 : 145, 48 ; 1929: 147, 288; 1930: Przemyst eherniczny, 201; 1931.

\section{Nature of Liquids.}

Prof. G. W. Stewart ${ }^{1}$ and Prof. E. N. da C. Andrade ${ }^{2}$ have agreed, one in the light of $\mathrm{X}$-ray diffraction experiments, and the other in the light of theoretical considerations of viscosity, that a liquid is, especially near the melting point, more akin to a solid than a gas.

It is perhaps worth while to point out that this view is consistent with what is observed concerning the specific heats of elements in the solid and liquid phases near the melting point. It is, in fact, the case that for the majority of monatomic elements hitherto investigated, the atomic heat of the liquid at constant volume is within about ten per cent of the value for the solid immediately below the melting point, and that this value is approximately $3 N_{0} k$ (that is, $3 R$ ), where $N_{0}$ is Avogadro's number and $k$ is Boltzmann's constant.

The value $3 R$ for the solid may be explained in terms of the three degrees of freedom of an atom, each degree of freedom contributing on the average $\frac{1}{2} k T$ to the energy as kinetic energy, together with (if the oscillation is simple harmonic in character) a further $\frac{1}{2} k T$ as potential energy.

The fact that the variation of energy content with temperature in the liquid is approximately the $\operatorname{sam} \theta$ as in the solid, is consistent with the view that in a liquid, at temperatures not too far above its melting point, the atoms continue to execute motions which may be described as approximately simple harmonic, the contribution per degree of freedom of the potential energy to the total being still approximately equal to that of the kinetic energy, which, since it depends on squares of velocities, must have the equipartition value of $\frac{1}{2} k T$.

If, on the other hand, the potential energy of atomic motion in a liquid were negligible compared with the kinetic energy, the atomic heat at constant volume would fall to the value appropriate to a monatomic gas, namely, $\frac{3}{2} R$. L. G. CARPENTER.

Physical Laboratory, University College, Southampton,

Dec. 3.

1 NATURE, 128, 727, Oct. 24, 1931

2 NATURE, 128, 835, Nov. 14, 1931

\section{Freshwater Eels in British India.}

For some ten years now I have been collecting data regarding the occurrence or absence of freshwater eels-of the genus Anguilla-in the fresh waters of the world, and we seem on the whole to have cleared up this matter. Yet there are two regions where some uncertainty still reigns and, curiously enough, British India is one of them.

I have seen specimens of freshwater eels from the greater part of India, from Burma to Bombay, but whether they occur north of Bombay, and especially in the River Indus, I have not succeeded in determin. ing, neither through the available literature nor through questions. Yet it would be astonishing, it seems to me, if there were no information on this subject from one of the principal river systems of the old World.

The question is of considerable importance for the understanding of the distribution of the freshwater eels, which spawn in the Indian Ocean. It would be of interest, therefore, if Indian zoologists, or others concerned with the fisheries there, could take up this question and discover whether freshwater eels of the genus Anguilla are really present or wanting in the River Indus.

Carlsberg Laboratorium,

Copenhagen, Denmark. JoHs. SCHMIDT.

\section{The Raman Band of Water.}

THE peculiar feature of the Raman band of water which I observed during my experimental researches ${ }^{1}$ may be produced, or at least essentially modified, by a particular and unexpected action of the spectro. graph.

The Hilger $E 1$ spectrograph used by me being provided, immediately behind the slit, with a total. reflecting prism of crystalline quartz, that is, with a double-refracting system, we are making use of an optical arrangement that may cause interferential phenomena, if the incident light is even partially polarised.

Under such conditions the use of the above-men. tioned spectrograph is unadvisable. G. Bolla.

Physical Institute of the Royal University, Milan, Dec. 10.

NATURE, Sept. 26, 1931.

No. 3245, VoL. 129] 